\title{
A Rapid and Highly Efficient Genotyping Pipeline for Screening Gene Targeted Mouse Embryonic Stem Cells
}

\section{Roger Caothien}

Genentech Inc

Charles Yu

Genentech Inc

Lucinda Tam

Genentech Inc

Robert Newman

Genentech Inc

\section{Brian Nakao}

Genentech Inc

\section{Tuija Alcantar}

Genentech Inc

\section{Natasha Baccaro}

Genentech Inc

Juan Reyes Jr

Genentech Inc

Anna Pham

Genentech Inc

Merone Roose-Girma ( $\nabla$ girma.meron@gene.com )

Genentech Inc https://orcid.org/0000-0003-1601-571X

\section{Research Article}

Keywords: ES cells, Gene targeting, Genotyping, Magnetic activated cell sorting MACS, Multiplex digital PCR ddPRC, Transgenic mice

Posted Date: July 30th, 2021

DOI: https://doi.org/10.21203/rs.3.rs-748320/v1

License: (c) (1) This work is licensed under a Creative Commons Attribution 4.0 International License. 



\title{
A Rapid and Highly Efficient Genotyping Pipeline for Screening Gene Targeted Mouse Embryonic Stem Cells
}

Roger Caothien, Charles Yu, Lucinda Tam, Robert Newman, Brian Nakao, Tuija Alcantar, Natasha Bacarro, Juan Reyes Jr, Anna Pham, Merone Roose-Girma

Department of Molecular Biology, Genentech, Inc., South San Francisco, California, USA. Corresponding author: Merone Roose-Girma

girma.meron@gene.com

Orcid IDs

Roger Caothien: 0000-0001-6286-7767

Lucinda Tam: 0000-0002-3101-6684

Merone Roose-Girma: 0000-0003-1601-571X

\begin{abstract}
:
Gene targeting in mouse ES cells replaces or modifies genes of interest; conditional alleles, reporter knock-ins, and amino acid changes are common examples of how gene targeting is used. For example, enhanced green fluorescent protein or Cre recombinase is placed under the control of endogenous genes to define promoter expression patterns. The most important step in the process is to demonstrate that a gene targeting vector is correctly integrated in the genome at the desired chromosomal location. The rapid identification of correctly targeted ES cell clones is facilitated by proper targeting vector construction, rapid screening procedures, and advances in cell culture. The addition of magnetic activated cell sorting (MACS) technology and multiplex droplet digital PCR (ddPCR) to the ES cell screening process can achieve a greater than $60 \%$ assurance that ES clones are correctly targeted. In a further refinement of the process, drug selection cassettes are removed from ES cells with adenovirus technology. This improved workflow reduces the time needed to generate preclinical animal models. Faster access to animal models for therapeutic target identification and experimental validation can accelerate the development of therapies for human disease.
\end{abstract}


Consent to participate: Not Applicable

Consent for publication: Not Applicable

\section{Keywords:}

ES cells

Gene targeting

Genotyping

Magnetic activated cell sorting MACS

Multiplex digital PCR ddPRC

Transgenic mice

\section{Declarations:}

Funding: This work was supported by Genentech Inc.

Conflicts of interest: All authors are or were employed by Genentech during the course of these studies and have held equity in the Roche group.

Availability of data and material: Not Applicable

Code availability: Not Applicable

Authors' contributions: Not Applicable

Ethics approval All experiments were conducted in accordance with the National Institutes of Health Guidelines for the Care and Use of Mice in Research and were approved by the Genentech Institutional Animal Care and Use Committee. All mice were housed and bred under specific pathogen-free conditions.

Consent to participate: Not Applicable

Consent for publication: Not Applicable

\section{Introduction:}

Gene targeting by homologous recombination between targeting vectors and chromosomes in embryonic stem cells (ES cells or ESCs) has been widely used in biomedical research (Capecchi 2005). This technology has been used to label genes and 
cells, for instance with enhanced green fluorescent protein (EGFP), to report expression patterns and follow expression during embryonic development (Todaro et al. 2019). ES cell technology has also been used to produce conditional knockout (CKO) gene mouse models (Le and Sauer 2001, Nagy 2000, Skarnes et al. 2011). To produce CKO alleles, loxP sites are first placed around critical exons in gene targeting vectors (Skarnes et al., 2011, Newman et al., 2015). Homologous recombination between the vector and the targeted gene replaces the wild type exon with the floxed exon so that the gene is normally expressed until Cre recombinase-mediated recombination removes the exon from the gene, causing a loss of gene function. For correct function of an EGFP reporter gene or CKO genes, it is essential to use precise screening methods that will discriminate between random integration of gene targeting vectors and correctly targeted insertion of experimental elements.

Producing genetically engineered mice from ES cells requires electroporation of ES cells with a targeting vector (Saunders 2011) and genotyping the ESCs with a screen to identify correct gene-targeting events (Fig. 1). One screening method utilizes long-range PCR, enabling identification of ES cells that carry correctly targeted genes (Friedel 2009; Skarnes et al. 2011). To achieve this, primers are placed within the targeting vector (for example. in the drug selection cassette) and outside the homology arms of the targeting vector. An amplicon will only result if the gene of interest is correctly targeted. We have used a long-range PCR to screen for correctly targeted mouse ES clones with modest success. We find the process to be timeconsuming, and the success rate is low and often requires optimization. For this reason, we developed a highly efficient genotyping platform to improve the screening process. We replaced long range PCR screens with a pipeline that includes Magnetic Activated Cell Sorting (MACS) and adapted the loss of native allele assay (LOA) (Valenzuela et al. 2003) to our multiplex droplet digital PCR (ddPCR) screening process (Fig. 1). ES cells that carry one copy of the native gene instead of two are likely to carry a correctly targeted gene. ES cells that carry two copies of the gene of interest indicate that the drug resistant cells carry random vector integrations instead of targeted integrations. We also use ddPCR to test for the presence of the Y chromosome. Mouse ES cells are XY and the loss of the Y chromosome during cell culture will reduce the efficiency of germline transmission of the targeted gene. ddPCR accurately quantifies targeted nucleic acid sequences by randomly partitioning sample DNA into isolated droplets, so that most droplets contain one template (Bell et al. 2018). The template within each droplet is amplified and detected in a sequence-specific manner with a hydrolysis probe. Different targets, such as neo or Y specific sequences, may be quantified with uniquely labeled probes.

Feeder cells are widely used in the culture of mouse ES cells and human pluripotent stem cells (IPSC). Feeder cells support the maintenance of ES cell and iPSC pluripotency by secreting extracellular matrix molecules and cytokines enabling robust 
expansion of ESCs and iPSCs (Smith and Hooper 1987; Takahashi et al. 2009). Feeder-cell derived DNA in mouse ES cell cultures present a confounding factor during DNA screening. We describe the application of magnetic cell separation (MACS, Baskale, D. et al. 2012) to eliminate feeder cells and their DNA from ES cell DNA preparations. We validated feeder-free DNA preparations in our ddPCR LOA assays to screen for correctly targeted ES cells. We find that MACS effectively removes contaminating DNA and provides accurate ddPCR results.

In gene targeting vectors, a drug selection cassette such as PGKneo (Tybulewicz et al. 1991) is placed next to a reporter gene such as a fluorescent protein coding sequence (Tian et al. 2011) or next to one of the loxP sites in conditional knockout vectors (Newman et al. 2015) to enrich for correctly targeted ES cells. To achieve normal gene expression, it is essential to remove the PGKneo cassette from the genome; otherwise, the bidirectional PGK promoter may disrupt the expression of the targeted gene and/or neighboring genes (Meyers 1998; Nagy et al. 1998; Scacheri et al. 2001; Pan et al. 2016). Conventional approaches to remove FRT-flanked PGKneo cassettes rely on breeding FRT-carrying mice with FLP recombinase-expressing mice (Kranz et al. 2010) or on treating ES cells with cell-permeable FLP recombinase (Patsch et al. 2010) or FLPoexpressing plasmids (Raymond and Soriano 2007).

Here, we describe a novel and effective approach to the removal of FRT-flanked drug selection cassettes: adenovirusmediated delivery of FLPo recombinase directly into ES cells. This ES cell treatment dramatically accelerates the process of removing drug selection cassettes from gene-targeted ES cells. In comparison to conventional methods, we find that the screening workflow of MACS, dPCR, and adenovirus FLPo recombinase delivery combined, dramatically reduces the time required to identify correctly targeted ES cells.

\section{Methods}

\section{Mouse ES Cell Culture and Transfection}

C57BL/6N C2 ES cells (Gertsenstein et al. 2010) were cultured on mitotically inactivated primary mouse embryonic fibroblasts (MEFs) in ES cell medium: DMEM, high glucose, no glutamine (Gibco catalog no. 11960077) supplemented with 15\% FBS pre-tested for ES cell culture as described (Hughes and Saunders 2011), 2 mM glutamine (Gibco catalog no, A2916801), 0.1 mM NEAA (Gibco catalog no. 11140050), 0.1 mM 2- mercaptoethanol (MilliporeSigma catalog no M7522) and 1000U/ml ESGRO (MilliporeSigma catalog no ESG1107). ESC transfections with gene targeting vectors were performed as previously described (Hughes and Saunders 2011). ES cells were plated and placed under drug selection. After 10 days of selection, as many as 96 ES cell clones with normal ES cell morphology were picked and cultured on two 2 96- 
well plates. The master plate was frozen and later used to recover gene-targeted clones. The second plate was used to grow clones for DNA extraction and DNA screening to identify clones that carry correctly targeted genes.

\section{Adenovirus Transfection}

Gene-targeted ES cells were cultured for 2 days on feeders in 24-well plates containing ES cell medium. On the day of virus treatment, medium is aspirated, and cells are washed with $2 \mathrm{ml}$ PBS. $300 \mu \mathrm{l}$ of $0.25 \%$ trypsin-EDTA is added to the cells and the plate is placed in a $37^{\circ} \mathrm{C}, 5 \% \mathrm{CO} 2$, humidified incubator. After 10 minutes of incubation, the trypsinized cells are pipetted with a Pipetman P1000 pipet (Gilson catalog no. FA10006M) to form a single-cell suspension and transfer it to a centrifuge tube. The cell suspension is centrifuged for $5 \mathrm{~min}$ at $400 \mathrm{xg}$. The supernatant is aspirated, and the cells are re-suspended in 3 $\mathrm{ml}$ of ES cell media containing 2\% FBS. Trypan blue staining is used to count cells with a hemocytometer. Wells of a 24well feeder plate were filled with $5 \times 10^{4}$ cells in $0.5 \mathrm{ml}$ of ES media with $2 \%$ FBS immediately prior to virus treatment. Frozen adenovirus preparation Ad(RGD)- CMV-FLPo (Vector Biolabs) is thawed for 2 minutes. Virus with an initial titer of $1 \mathrm{X} 10^{10} \mathrm{PFU} / \mathrm{ml}$ (10ul) is added to the $5 \mathrm{X} 10^{4}$ cells to achieve a multiplicity of infection (MOI) of 1000 . Cells and viruses are mixed by pipetting once with a $\mathrm{P} 1000$ and placed in a $37^{\circ} \mathrm{C}, 5 \% \mathrm{CO} 2$, humidified incubator. 24 hours later, medium is replaced with fresh ES cell medium containing 15\% FBS. Cells are washed with PBS 48 hours after virus addition and trypsinized in $300 \mu 1$ of $0.25 \%$ trypsin-EDTA for 10 minutes in a $37^{\circ} \mathrm{C}, 5 \% \mathrm{CO} 2$, humidified incubator. After trypsinization, virus-treated cells are pipetted with a P1000 to form a single-cell suspension. A P200 pipette was used to transfer $25 \mu 1$ (6.25\%) or $50 \mu \mathrm{l}(12.5 \%)$ of the trypsinized cells to a $10 \mathrm{~cm}$ diameter feeder plate containing fresh ES cell medium supplemented with $15 \%$ FBS. Plates are rocked to disperse the cells and place them in the incubator. ES cell medium, 15\% FBS, is changed daily for 6 days. ES cell clones are picked and grown in duplicate 96-well feeder plates as described above.

\section{MultiMACS Feeder Cell Removal:}

96-well plates containing mouse ES cells and feeder cells were washed once with 200ul of PBS. PBS is aspirated and replaced with $40 \mathrm{ul}$ of $0.25 \%$ trypsin-EDTA. Cells are placed in a $37^{\circ} \mathrm{C}, 5 \% \mathrm{CO} 2$ humidified incubator for 20 minutes. Trypsinized cells are pipetted 10 times to produce a single cell suspension. After adding 150ul of ES cell medium, 10ul of feeder removal microbeads are added (Miltenyi Biotec catalog no. 130-095-531) and resuspended by gentle pipetting. The mixture is then incubated for 20 minutes at room temperature on an orbital shaker (300rpm). Following incubation, the suspension is loaded on to a 96-well column (Miltenyi Biotec catalog no. 130-092-445). The ES cell-containing flow-through is collected while the feeder cells remain in the column. The collected ES cells are spun down in a centrifuge at 3,000rpm and genomic DNA in 96-well plate format was prepared as described (Ramírez-Solis et al. 1992). 


\section{Multiplex Digital PCR}

The BioRad QX200 AutoDG ddPCR system is used to determine the PGKneo gene copy number and Y chromosome copy number. A ddPCR assay for the neomycin gene is used to confirm correct gene targeting and also used to confirm the elimination of the FRT-flanked PGKneo cassette from ES cell clones. An assay for the mouse Uty gene (ubiquitously transcribed tetratrico-peptide repeat containing, Y-linked) is used to count the number of Y chromosomes. DNA from ES clones previously shown to carry 1 or 0 copies of PGKneo and the Y chromosome is used as positive and negative control, respectively. ddPCR is performed by mixing 20ng of genomic ES cell DNA with 10ul Taq Mastermix (BioRad catalog no.186-3024), 900nm Primer1, 900nm Primer 2, etc. in a volume of $25 \mathrm{ul}$ with $250 \mathrm{~nm}$ labeled neo probe and $250 \mathrm{~nm}$ of labeled Uty probe. Samples are loaded onto a BioRad QX200 instrument. Results are obtained and collated.

\section{Results}

\section{Long Range PCR}

Long range PCR was used to detect correct targeted integration for 45 genes. One primer was anchored in the PGKneo cassette and the other primer was placed outside an arm of homology so that only correctly targeted genes would amplify. We found that the overall gene targeting efficiency was $2.4 \%$ of clones that were screened (Table 1 ). This compares favorably to other reports (Skarnes et al. 2011). Compared to the ddPCR process, this method is time-consuming; and the usual time to identify targeted ES cells was four weeks.

\section{ddPCR}

We used ddPCR to detect correct gene targeting (loss of allele assay (LOA) and Y chromosome assay) and to confirm the elimination of the FRT flanked PGKneo cassette from ES cell clones after adenovirus delivery of FLP recombinase to ES cells. LOA are quantitative ddPCR assays that detect sequences in the targeting vector to determine the copy number of the desired change (genomic deletion, sequence insertion, or sequence replacement). For a gene that is targeted correctly, the LOA assay will detect one copy of the targeted gene and one copy of the wild type gene. In random integration events the wild type sequence is not disrupted and the LOA detects two copies of the wild type gene (Fig. 2). Results from ten gene targeted experiments showed that overall, $4.0 \%$ of clones were targeted (Table 2). The assay for the Uty gene on the Y chromosome (ubiquitously transcribed tetratrico-peptide repeat containing, Y-linked) showed that fewer than 0.1\% of ES clones lost the Y-chromosome (data not shown). After adenovirus delivery of FLP recombinase to remove the PGKneo cassette from 324 ES cell clones, we found that 158 clones (48.8\%) had indeed lost PGKneo (Table 3). We found that the 
usual time needed to detect targeted ES cells by multiplexed ddPCR was 3 days. The simultaneous detection of the Y chromosome and use of ddPCR to show that the FRT flanked drug selection was removed also reduced the time needed to characterize targeted ES cells.

\section{Adenovirus FLP-Mediated PGKneo Excision}

We treated ES cells with an Ad(RGD)-CMV-FLPo adenovirus to remove FRT-flanked PGKneo cassettes. After Ad(RGD)CMV-FLPo treatment, FLPo/FRT mediated recombination will recombine the 2 FRT sites, remove the intervening sequence, and leave a single FRT site resulting in a CKO allele (Figure 4). To select an effective adenovirus treatment, taking into consideration effects on cell morphology and toxicity, we treated ES cells with adenovirus preparations that delivered MOIs of $250,500,1000$, or 5000 . We determined that a 1000 MOI was ideal for producing viable cells that had lost the PGKneo cassette. ddPCR analysis of ES cells treated with 1000 MOI showed that $48.8 \%$ clones were PGKneo negative (Table 3).

\section{Discussion}

We applied an automated genotyping pipeline to the identification of gene-targeted ES cells that can be used to produce mouse models for drug discovery and basic biomedical research. The screening process included MACs feeder cell removal, ddPCR loss of allele assays, and adenovirus delivery of FLPo recombinase. Feeder cell removal is a critical step to eliminate contamination of wild type genomic DNA from ES cell DNA samples committed to ddPCR assays. The use of multiplexed ddPCR assays to detect loss of allele, $\mathrm{Y}$ chromosome copy number, and drug selection copy number accelerated the discovery of correctly targeted ES cells. Delivery of FLPo recombinase to ES cells by adenovirus was efficient. Screening showed that adenovirus treatment removed the FRT-flanked drug selection cassette from half of the ES cell clones.

Conventional screening assays that use long-range PCR and DNA sequencing or Southern blot analysis to identify targeted ES cells are more laborious and time consuming than the MACs/ddPCR pipeline described here. Importantly, multiplexed LOA ddPCR allows for the simultaneous detection of the Y chromosome and PGKneo copy number, unlike long range PCR. Direct treatment of targeted ES cell clones in cell culture to remove FRT-flanked drug selection cassettes eliminated $a$ priori the need to establish a mouse colony from ES cells and then to use in vivo breeding of mice with FLPo transgenic mice or to produce mouse zygotes for in vitro treatment with FLPo recombinase.

Culture of pluripotent mouse ES cells on MEF cells contributes to the maintenance of the most valuable characteristic of ES cells: the ability to contribute to germ cells (sperm) and thus to transform genetic modifications engineered in ES cells to animals carrying experimental genetic modifications (Capecchi 2005). However, co-purification of MEF DNA when ES cell 
DNA is prepared interferes with downstream screening. Conventional approaches to remove MEFs from ES cells include removing MEFs by serial culture in the absence of MEFs (Nagy et al. 1993), differential sedimentation, or pre-plating to separate MEFs from ES cells ( $\mathrm{Li}$ et al. 2008). Here we demonstrate that a rapid (less than 30 minutes) MACS application to remove MEFs from culture is superior to prior methods used to obtain pure populations of mouse ES cells for DNA extraction.

Long range PCR or Southern blotting can be employed to screen for targeted mutation created by the introduction of the targeting vector (Friedel 2009; McMahon and Bradley 1990; Saunders 2011; Skarnes et al. 2011). The purpose of this method is to demonstrate that the gene targeting vector is correctly inserted in the genome or correctly replaced by genomic DNA sequences. Southern blotting takes advantage of changes in restriction enzyme maps in the genome (Friedel 2009; Saunders 2011). This approach has largely given away to PCR screens that position primers in the genomic DNA outside of the gene targeting vector and within the targeting vector in such a way that an amplicon is produced only when the vector has been incorporated in the correct location (Skarnes et al. 2011). Long range PCR was incorporated into the workflow to screen for correctly targeted ES clones with high success (Table 1). However, long range PCR of $4000 \mathrm{bp}$ or more of genomic DNA is not always efficient and presents technical challenges. We recently replaced the long-range PCR with ddPCR. This modification allows us to take advantage of LOA assays to detect correct gene targeting (Valenzeuela et al. 2003). We successfully employed LOA to identify correctly targeted ES cells (Table 2). We found that ddPCR is much faster and reliable than long range PCR and DNA sequencing.

Ad(RGD)-CMV-FLPo treatment effectively removes FRT-flanked PGKneo cassettes from targeted genes in ES cells. It is essential to remove the PGKneo drug selection cassette to prevent confounding phenotypes in mouse lines produced from gene-targeted ES cells. Conventional approaches to remove PGKneo cassettes involve transfection of ES cells with plasmids that express FLPo (Raymond and Soriano 2007). Other methods to remove the cassette depend on producing gene-targeted mice from ES cells. In these methods, the mice are mated with FLPo transgenic mice to remove the FRT-flanked PGKneo cassette (Birling et al. 2012, Kranz et al. 2010). Alternatively, the mice can be used to produce gene-targeted zygotes that are microinjected with FLP recombinase (Ludwig et al. 1996) or are treated with cell-permeable FLP recombinase (Patsch et al. 2010). In practice, zygote treatment methods are not routinely used in the field. We find that Ad(RGD)-CMV-FLPo treatment is rapid and effective. We observed that up to $67 \%$ of ES cells treated with Ad(RGD)-CMV-FLPo underwent recombination. It is likely that the location of the FRT sites in the genome and the surrounding DNA affects the efficiency of recombination in a locus specific fashion. These effects are seen as variations in the efficiency of FLPo-mediated recombination, as has been 
reported for Cre recombinase (Liu et al. 2013).

One of the major challenges in the generation of genetically engineered mice from ES cells is the multi-step process to screen large numbers of ES cell clones for gene targeting events. To address this challenge, we report here for the first time the development and application of a rapid screening pipeline that combines MACS, ddPCR assays, and adenovirus-mediated FLPo recombinase delivery. The principal advantage of our genotyping pipeline is rapid high capacity screening of large numbers of mouse ES clones in a flexible, low cost, and easy-to-use process. We have successfully identified over 100 genetargeted ES cell clones across multiple projects. Our pipeline delivers ES cells ready for blastocyst microinjection and mouse model production. Our screening pipeline has the potential to facilitate and accelerate screening protocols not only for mouse ES cells but also for the screening of engineered cell lines for the $\mathrm{p}$

\section{Acknowledgements}

We thank Genentech Sequencing/NGS Lab and BioMolecular Resources (BMR) for high quality DNA preparation. We thank Thom Saunders for helpful discussion. We thank Andrew Peterson and Soren Warming for their departmental support.

\section{References}

Baskale, D. et al. (2012) Use of magnetically enriched pluripotent stem cells increases chimerism rate after blastocyst injection and enables the use of inbred ES cell lines for tetraploid complementation. MACS\&more 14 (2). https:// www.miltenyibiotec.com

Bell AD, Usher CL, McCarroll SA (2018) Analyzing Copy Number Variation with Droplet Digital PCR. Methods Mol Biol. 1768:143-160. doi: 10.1007/978-1-4939-7778-9_9.

Birling MC, Dierich A, Jacquot S, Hérault Y, Pavlovic G (2012) Highly efficient, fluorescent, locus directed cre and FlpO deleter mice on a pure C57BL/6N genetic background. Genesis. 50:482-9. doi: 10.1002/dvg.20826

Capecchi MR (2005) Gene targeting in mice: functional analysis of the mammalian genome for the twenty-first century. Nat Rev Genet 6:507-12. doi: 10.1038/nrg1619 
Gertsenstein M, Nutter LM, Reid T, Pereira M, Stanford WL, Rossant J, Nagy A (2010) Efficient generation of germ line transmitting chimeras from C57BL/6N ES cells by aggregation with outbred host embryos. PLoS One. 5:e11260. doi: 10.1371/journal.pone.0011260.

Hughes ED, Saunders TL (2011) Gene Targeting in Embryonic Stem Cells. In: Pease S and Saunders TL (eds) Advanced Protocols for Animal Transgenesis: An ISTT Manual. Springer-Verlag, Berlin. Pp. 291-325.

Kranz A, Fu J, Duerschke K, Weidlich S, Naumann R, Stewart AF, Anastassiadis K (2010) An improved Flp deleter mouse in C57Bl/6 based on Flpo recombinase. Genesis 48:512-20. doi: 10.1002/dvg.20641

Le Y, Sauer B. (2001) Conditional gene knockout using Cre recombinase. Mol Biotechnol. 17:269-75. doi: 10.1385/MB:17:3:269.

Li Z, Barron MR, Lough J, Zhao M (2008) Rapid single-step separation of pluripotent mouse embryonic stem cells from mouse feeder fibroblasts. Stem Cells Dev 17:383-7. doi: 10.1089/scd.2007.0138

Liu J, Willet SG, Bankaitis ED, Xu Y, Wright CV, Gu G. 2013. Non-parallel recombination limits Cre-LoxP-based reporters as precise indicators of conditional genetic manipulation. Genesis. 51:436-42. doi: 10.1002/dvg.22384

Ludwig DL, Stringer JR, Wight DC, Doetschman HC, Duffy JJ (1996) FLP-mediated site-specific recombination in microinjected murine zygotes. Transgenic Res. 5:385-95. doi: 10.1007/BF01980203

McMahon AP, Bradley A (1990) The Wnt-1 (int-1) proto-oncogene is required for development of a large region of the mouse brain. Cell 62:1073-85. doi: 10.1016/0092-8674(90)90385-r

Meyers EN, Lewandoski M, Martin GR (1998) An Fgf8 mutant allelic series generated by Cre- and Flp-mediated recombination. Nat Genet. 18:136-41. doi: 10.1038/ng0298-136

Nagy A, Moens C, Ivanyi E, Pawling J, Gertsenstein M, Hadjantonakis AK, Pirity M, Rossant J (1998) Dissecting the role of 
N-myc in development using a single targeting vector to generate a series of alleles. Curr Biol 8:661-4. doi: 10.1016/s09609822(98)70254-4

Nagy A (2000) Cre recombinase: the universal reagent for genome tailoring. Genesis 26:99-109.

Nagy A, Rossant J, Nagy R, Abramow-Newerly W, Roder JC (1993) Derivation of completely cell culture-derived mice from early-passage embryonic stem cells. Proc Natl Acad Sci U S A. 90:8424-8. doi: 10.1073/pnas.90.18.8424

Newman RJ, Roose-Girma M, Warming S (2015) Efficient conditional knockout targeting vector construction using coselection BAC recombineering (CoSBR). Nucleic Acids Res 43:e124. doi: 10.1093/nar/gkv600

Pan Y, Zhang L, Liu Q, Li Y, Guo H, Peng Y, Peng H, Tang B, Hu Z, Zhao J, Xia K, Li JD (2016) Insertion of a knockoutfirst cassette in Ampd1 gene leads to neonatal death by disruption of neighboring genes expression. Sci Rep 6:35970. doi: $10.1038 / \operatorname{srep} 35970$.

Patsch C, Peitz M, Otte DM, Kesseler D, Jungverdorben J, Wunderlich FT, Brüstle O, Zimmer A, Edenhofer F (2010) Engineering cell-permeant FLP recombinase for tightly controlled inducible and reversible overexpression in embryonic stem cells. Stem Cells. 28:894-902. doi: 10.1002/stem.417.

Ramírez-Solis R, Rivera-Pérez J, Wallace JD, Wims M, Zheng H, Bradley A (1992) Genomic DNA microextraction: a method to screen numerous samples. Anal Biochem 201:331-5. doi: 10.1016/0003- 2697(92)90347-a

Raymond CS, Soriano P (2007) High-efficiency FLP and PhiC31 site-specific recombination in mammalian cells. PLoS One. 2:e162. doi: 10.1371/journal.pone.0000162.

Saunders TL (2011) Gene Targeting Vector Design for Embryonic Stem Cell Modifications. In: Pease S and Saunders TL (eds) Advanced Protocols for Animal Transgenesis: An ISTT Manual. Springer-Verlag, Berlin. pp. 57- 79

Scacheri PC, Crabtree JS, Novotny EA, Garrett-Beal L, Chen A, Edgemon KA, Marx SJ, Spiegel AM, Chandrasekharappa SC, Collins FS (2001) Bidirectional transcriptional activity of PGK-neomycin and unexpected embryonic lethality in 
heterozygote chimeric knockout mice. Genesis 30:259-63. doi: 10.1002/gene.1072

Skarnes WC, Rosen B, West AP, Koutsourakis M, Bushell W, Iyer V, Mujica AO, Thomas M, Harrow J, Cox T, Jackson D, Severin J, Biggs P, Fu J, Nefedov M, de Jong PJ, Stewart AF, Bradley A (2011) A conditional knockout resource for the genome-wide study of mouse gene function. Nature 474:337-42. doi: 10.1038/nature10163

Smith AG, Hooper ML (1987) Buffalo rat liver cells produce a diffusible activity which inhibits the differentiation of murine embryonal carcinoma and embryonic stem cells. Dev Biol 121:1-9. doi: 10.1016/0012-1606(87)90132-1

Takahashi K, Narita M, Yokura M, Ichisaka T, Yamanaka S (2009) Human induced pluripotent stem cells on autologous feeders. PLoS One 4:e8067. doi: 10.1371/journal.pone.0008067

Tian H, Biehs B, Warming S, Leong KG, Rangell L, Klein OD, de Sauvage FJ (2011) A reserve stem cell population in small intestine renders Lgr5-positive cells dispensable. Nature 478:255-9. doi: 10.1038/nature10408

Todaro F, Campolo F, Barrios F, Pellegrini M, Di Cesare S, Tessarollo Ln, Rossi P, Jannini EA, Dolci S. (2019) Regulation of Kit Expression in Early Mouse Embryos and ES Cells. Stem Cells 37:332-344. doi: 10.1002/stem.2960.

Tybulewicz VL, Crawford CE, Jackson PK, Bronson RT, Mulligan RC (1991) Neonatal lethality and lymphopenia in mice with a homozygous disruption of the c-abl proto-oncogene. Cell 65:1153-63. doi: 10.1016/0092- 8674(91)90011-m

Valenzuela DM, Murphy AJ, Frendewey D, Gale NW, Economides AN, Auerbach W, Poueymirou WT, Adams NC, Rojas J, Yasenchak J, Chernomorsky R, Boucher M, Elsasser AL, Esau L, Zheng J, Griffiths JA, Wang X, Su H, Xue Y, Dominguez MG, Noguera I, Torres R, Macdonald LE, Stewart AF, DeChiara TM, Yancopoulos GD (2003) High-throughput engineering of the mouse genome coupled with high-resolution expression analysis. Nat Biotechnol 21:652-9. doi: 10.1038/nbt822. 
Table 1: Summary information on 4 of 45 targeted alleles screened in ES cells by long range PCR.

\begin{tabular}{|c|c|c|c|}
\hline Gene Name & Accession no. & Area of Focus & Targeting \\
Percentage (\%)
\end{tabular}


Table 2: Summary information on 4 of 10 targeted alleles in ES cells screened by ddPCR:

\begin{tabular}{|c|c|c|c|c|}
\hline Gene Name & Accession no. & Area of Focus & Homologous Arm & Positive \\
& & & Size (kb) & Identified by \\
& & & & ddPCR (\%) \\
\hline CHRNA9 & NM_001081104.1 & Neurobiology & 1.8 & 3 \\
\hline SGK1 & NM_011361.4 & Kinase & 1.87 & 3 \\
\hline GPCR5 & & & & \\
& & & & \\
\hline FLT13 & NM_010229.2 & Kinase & & \\
& & & & \\
& & & & \\
\hline
\end{tabular}


Table 3: Summary information on removal of FRT flanked PGKneo from ES cell clones treated with Ad(RGD)-CMV-FLPo in eight gene-targeting experiments.

\begin{tabular}{|c|c|c|c|}
\hline Experiment & Clones Picked & Clones with neo Flp out & $\%$ of Flipped \\
\hline 1 & 48 & 32 & 67 \\
\hline 2 & 48 & 25 & 52 \\
\hline 3 & 24 & 31 & 65 \\
\hline 4 & 48 & 13 & 27 \\
\hline 5 & 36 & 17 & 36 \\
\hline 6 & 48 & 11 & 23 \\
\hline 7 & 24 & 5 & 21 \\
\hline 8 & 48 & 24 & 50 \\
\hline
\end{tabular}




\section{Figure Captions:}

Fig. 1. Overview of Custom Mouse Creation. 1) After a gene of interest is identified and the desired genetic change is described, a gene targeting vector is cloned. a) The vector is electroporated into ES cells. Drug resistant clones are picked in duplicates. After ES cell proliferation in culture, ES cell clones are individually purified from feeder cells by MACS. DNA is extracted from individual ES cell clones and submitted to ddPCR analysis to identify correctly targeted clones. Duplicate clones known to be targeted are placed in culture and used for blastocyst microinjection to produce ES cell-mouse chimeras. The chimeras are bred to wild type mice for germline transmission to pups carrying the targeted gene.

Fig. 2 Loss of Allele Assay. ddPCR is performed with primers to a reference gene as a control for copy number. Primers to the gene of interest are designed to detect the wild type gene or the desired genetic change in the gene. In correctly targeted ES cells, the target wild type allele copy number is reduced to 1 from 2.

Fig. 3 Action of FLPo recombinase on an ES cell carrying a conditional gene targeting event. Vectors used to target ES cells include a PGKneo cassette for drug resistance selection that can interfere with normal gene expression. After ES cells are treated with Ad(RGD)-CMV-FLPo recombination between the FRT sites caused by FLPo recombinase removes the PGKneo cassette so that normal gene expression will occur. loxP: DNA sequence recognized by loxP sites. FRT: DNA sequence recognized by FLPo recombinase. PGKneo: Drug selection cassette used to enrich for targeted ES cells after electroporation. Probe: ddPCR probe used to detect PGKneo cassette. exon: Critical exon that will produce a frameshifted transcript that will cause nonsense-mediated mRNA decay and loss of protein expression. 


\section{Figures}

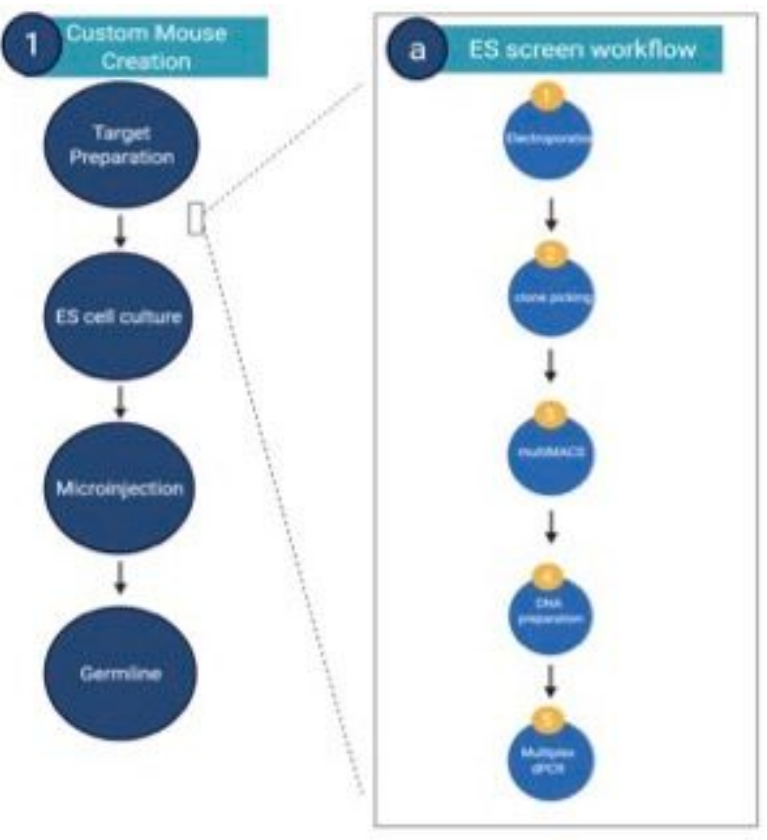

\section{Figure 1}

Overview of Custom Mouse Creation. 1) After a gene of interest is identified and the desired genetic change is described, a gene targeting vector is cloned. a) The vector is electroporated into ES cells. Drug resistant clones are picked in duplicates. After ES cell proliferation in culture, ES cell clones are individually purified from feeder cells by MACS. DNA is extracted from individual ES cell clones and submitted to ddPCR analysis to identify correctly targeted clones. Duplicate clones known to be targeted are placed in culture and used for blastocyst microinjection to produce ES cell-mouse chimeras. The chimeras are bred to wild type mice for germline transmission to pups carrying the targeted gene. 


\section{Reference gene (Two copies)}
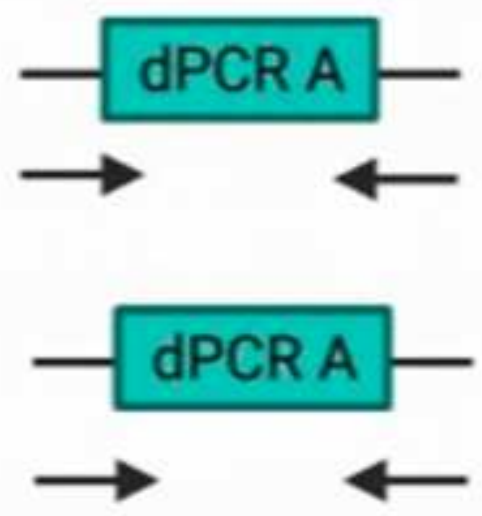

\section{Target gene \\ (One copy)}
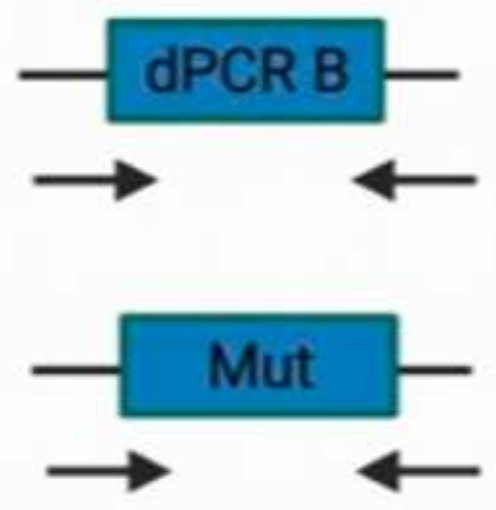

\section{Figure 2}

Loss of Allele Assay. ddPCR is performed with primers to a reference gene as a control for copy number. Primers to the gene of interest are designed to detect the wild type gene or the desired genetic change in the gene. In correctly targeted ES cells, the target wild type allele copy number is reduced to 1 from 2 .

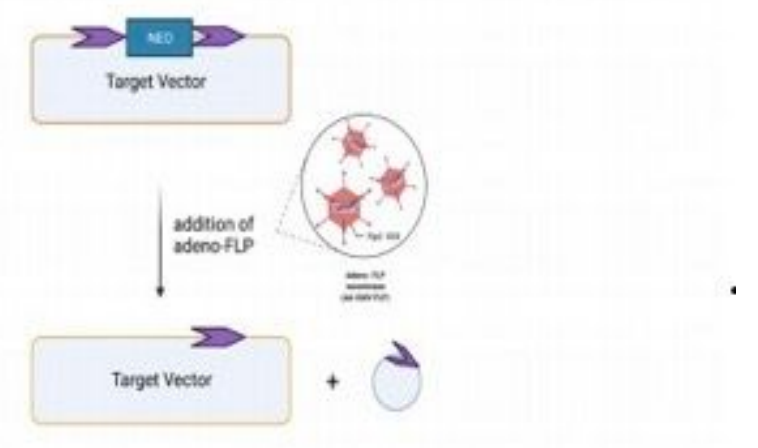

\section{Figure 3}

Action of FLPo recombinase on an ES cell carrying a conditional gene targeting event. Vectors used to target ES cells include a PGKneo cassette for drug resistance selection that can interfere with normal 
gene expression. After ES cells are treated with Ad(RGD)-CMV-FLPo recombination between the FRT sites caused by FLPo recombinase removes the PGKneo cassette so that normal gene expression will occur. loxP: DNA sequence recognized by loxP sites. FRT: DNA sequence recognized by FLPo recombinase. PGKneo: Drug selection cassette used to enrich for targeted ES cells after electroporation. Probe: ddPCR probe used to detect PGKneo cassette. exon: Critical exon that will produce a frameshifted transcript that will cause nonsense-mediated mRNA decay and loss of protein expression. 